\title{
Determining the best optimization method for large scale probabilistic supplier selection problem integrated with inventory management
}

\author{
Sutrisno Sutrisno*, Widowati Widowati, Sunarsih Sunarsih \\ Department of Mathematics, Diponegoro University, Semarang 50275 Indonesia
}

\begin{abstract}
In logistics and supply chain management, a problem of supplier selection is an optimization problem where the number of variables is growing exponentially which will produce a large-scale optimization problem. A right choice of the used method to solve is needed according to the performance of the method. This paper is considered to compare and analyse how the performance of some classic numerical optimization methods which are interior point, SQP, SQP-legacy and active-set to solve a large-scale optimization problem of a probabilistic supplier selection problem with inventory management. Word "probabilistic" in this case is referring to that the problem is involving some uncertain parameters approached by random variable (probabilistic parameter). We used the existing mathematical model of probabilistic supplier selection problem with inventory management provided in our previous works that only considering few numbers of decision variable then the occurred optimization problem is a small-scale problem that can be solved efficiently by analytical method or numerical method. Then, in this paper we resolved this model with huge number of decision variable indicated by the number of the supplier and time period that is large by using an existing numerical optimization method to analyse how the decision variable, is it reliable to be used or not. We generate some randomly data to simulate the problem and the results. From our computational experiment, the optimal decision variables obtained by the used methods are acceptable to be used as the decision that can be used to be applied by the decision maker. Based on the relative error given by these methods, the active set was given the best performance which means that active-set method is the best choice to solve.
\end{abstract}

\section{Introduction}

The most used approach to solve optimization problems in industrial process management is using mathematical optimization approach. There are thousands published research articles which were used a mathematical optimization approach to solve in many industrial fields like biopharmaceutical manufacturing [1,2], pig industry [3], automotive industry [4], sawmill industry [5] and many more. In industrial managerial optimization, supply chain optimization is one of the important components due that it is contributing significantly to the cost

* Corresponding author: $\underline{\text { s.sutrisno@live.undip.ac.id }}$ 
occurred in the process. Supply chain contains many parts from the upstream e.g. raw material supplier to the downstream e.g. end user/customer. To optimize these parts in one mathematical model, commonly it will be a very large model due to the huge of the variable number or function to be formulated. Therefore, researchers were developed the model in particular way for each part or few parts of them. One of these parts which is sufficiently significant in the contribution to the occurred cost is supplier selection process. It is a problem to find the best supplier(s) which will be contracted to supply some raw material(s) or product(s) [6].

Some mathematical model approaches to solve a supplier selection can be found in the literatures. Many of them were developed a model based on the specifications of the problem or assumptions which are used such as linear programming which is a sufficiently simple model containing a linear objective function and linear constraints occurred in the model [7], [8]. For a few advanced problem, a model was developed with considering negotiation process on multi-agent condition [9] and a decision making model with multi-objective approach [10]. Another important part in supply chain is inventory management which contributes to the holding cost and raw material/product stock management. The problem is determining the inventory level in the warehouse in order to minimize the holding cost occurred in the inventory system but the needed/demanded product volume is satisfied. For simple case, a mathematical optimization approach in a quadratic programming was developed to solve [11].

To solve an optimization problem computationally, most of the researchers or practitioners are used numerical method due to its superiority particularly on the computation speed. There are many algorithm alternatives that we can use, some of them are active-set, interior point, SQP (sequential-quadratic-programming) and SQP-legacy [12]. For interior point method, it is an old one but several researchers were developed it to improve the performance, for example, by preconditioning the problem [13], improving on the starting point [14], and modified interior-point called infeasible interior-point algorithm to solve stochastic complementarity problems [15], besides some application researches were conducted to show the superiority of this method e.g. for atomic norm soft thresholding [16]. Active-set method has also been developed to maintain its performance by modifying the warm-starting procedure in active-set algorithm [17]. The excellency of these methods can be found in some application research articles like in mechanical engineering [18, 19], machine learning [20], tomography application [21], hydrocarbon reservoir management [22], haptic display designing [23], and calculating the worst resonance response of nonlinear systems [24].

For a large supply chain problem, for instant problem with thousands of product types or supplier alternatives, the corresponding mathematical optimization model will be a largescale optimization problem. Fortunately, following the big data era and the massive developing on computer's capability, for now data is easier to get and some large computational problems can be handled. In this paper, we apply the interior point, SQP, SQPlegacy and active-set method to solve a large-scale optimization model numerically for supplier selection and inventory management problem solving considering random demand. This works is aimed to determine which method give the best performance in order to known the best choice from these methods that reasonable to be used to solve the problem in real life or real problem faced by the manager in manufacturer industries.

\section{Mathematical model}

In this discussion, we re-solve a problem in our previous work by using several algorithms described in the introduction. Let the notation $S$ is denoting the number of supplier 
alternatives, $X_{t s}$ is denoting the amount of the product purchased to supplier $s$ at time/review period $t, I_{t}$ is denoting the product stock level in the inventory at time/review period $t, U_{t, s}$ is denoting the cost needed to purchase one unit product to supplier $s$ at time/review period $t, H_{t}$ is denoting the cost needed to store one unit product along one time/review period $t$ and finally $E[\cdot]$ is denoting the expectation. The mathematical optimization model is described as follows [11]:

$$
\min \tilde{Z}=E[Z]=E\left[\sum_{t=1}^{T} \sum_{s=1}^{S} U_{t s} X_{t s}+\sum_{t=1}^{T} H_{t} I_{t}+\sum_{t=1}^{T}\left|I_{t}-r_{t}\right|\right]
$$

subject to:

$$
\begin{gathered}
I_{t-1}+\sum_{s=1}^{S} X_{t s}-I_{t} \geq \hat{D}_{t}, \forall t \in T ; \\
X_{t s} \leq C_{s}, \forall t \in T, \forall s \in S ; \\
I_{t} \leq M, \forall t \in T ; \\
X_{t s} \geq 0 \text { and integer, } \forall t \in T, \forall s \in S .
\end{gathered}
$$

The interpretation of this model is explained as follows. The model is minimizing the total cost expectation which contains total purchasing cost and total inventory cost for all time/review periods where the last objective function's term is representing the reference level tracking for inventory level. The constraints that should be hold in the model are representing the conditions in the problem. First, the demand value should be satisfied by the available product amount. If the notation for demand value is $\hat{D}_{t}$ (assumed to be a variable random due to the demand value is uncertain and this uncertainty is approached as a variable random with some known probability distribution) at time/review period $t$ then the constraint (2) should be hold. Second, each supplier has a maximum capacity to supply the product, then the amount of the purchased product should be not exceeding the supplier's capacity. If $C_{s}$ is denoting the maximum supplier's capacity for any supplier $s$, then the constraint (3) should be satisfied. Third, the warehouse has its maximum inventory level, hence the stored product should not exceed its maximum capacity. If $M$ is denoting the maximum warehouse's capacity, then constraint (4) should be occupied. Last, non-negativity and integer constraint (5) must be added to ensure that the product volume is integer and nonnegative. This optimization model is classified into constrained quadratic programming with random parameter

\section{Numerical experiment}

We have solved the model (1) by using interior point, SQP, SQP-legacy and active-set where the supplier alternatives are S1, S2 and S3. The used parameter values are described as follows: (1) purchase cost per unit product is $\$ 50$ for $\mathrm{S} 1, \$ 52$ for $\mathrm{S} 2$ and $\$ 50$ for $\mathrm{S} 3$, (2) Maximum supplying capacity is 300 units for S1, 500 units for S2 and 250 units for S3, (3) Maximum inventory level (unit) in the storage/warehouse is 300 units, (4) Cost to store one unit product for one time period is $\$ 4$. In this numerical experiment, these parameter values are constant to time/review period which it means that these values are the same to all 
time/review period. The remain parameter, i.e. demand value is assumed to be a random variable denoted by $D_{t}, t=1,2,3$ with the following probability density function:

$$
f_{D_{t}}(D)=\left\{\begin{array}{l}
0.3, \text { if } D=100 \\
0.3, \text { if } D=150 \\
0.4, \text { if } D=200
\end{array}\right.
$$

for any time/review period $t$. To solve the optimization computationally, we input the mathematical model in LINGO 18.0 software then we generate its deterministic equivalent optimization model to be exported into MPS file. Next, we import the MPS file into MATLAB software by using LINDO API programming language. Finally, we solve the problem using MATLAB Optimization Toolbox using daily used personal computer with i7 Octa Core $2.9 \mathrm{GHz}$ of Processor, $4 \mathrm{~GB}$ of RAM and Windows 10 of OS. The optimal decision results are illustrated in Figure 1 and Figure 2.

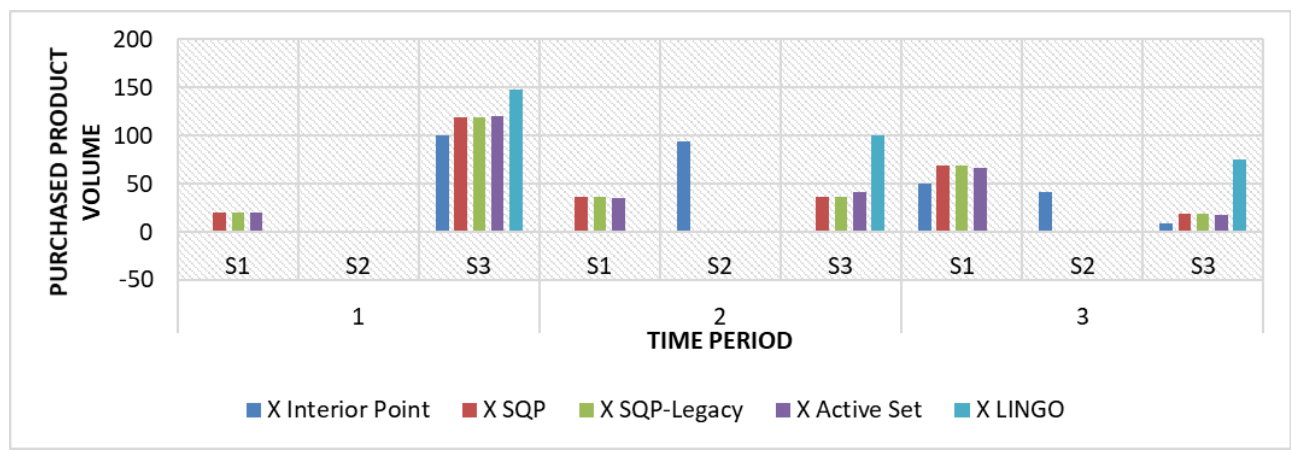

Figure 1. The optimal decision i.e. optimal purchasing product volume for scenario-1 generated by interior point, SQP, SQP-legacy, active-set and GRG (LINGO)

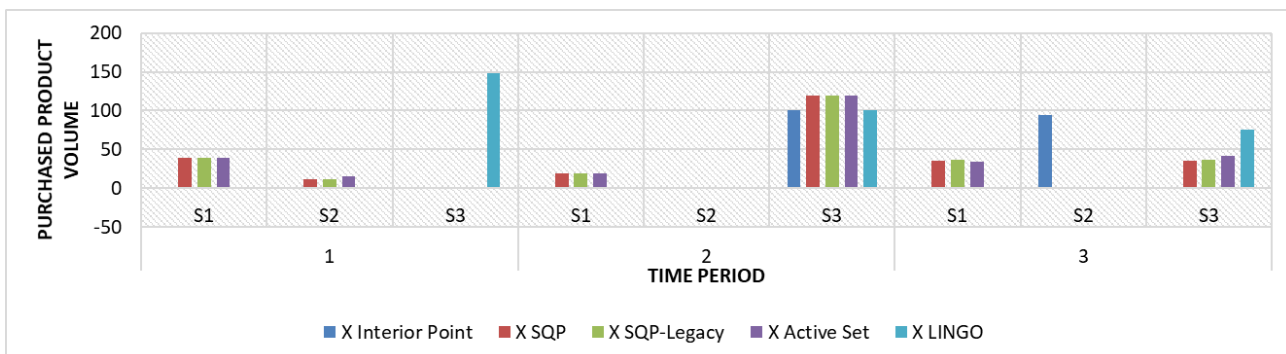

Figure 2. The optimal decision i.e. optimal purchasing product volume for scenario-2 generated by interior point, SQP, SQP-legacy, active-set and GRG (LINGO)

As described in Figure 1(a), for time/review period-1, the SQP, SQP-legacy and activeset method resulted around 44 units from S1, 100 units from S2, and 0 unit from S3 whereas GRG (LINGO) resulted around 150 units from S3 and 0 unit each from S1 and S2. These results show us that interior point, SQP, SQP-legacy and active-set were resulted the similar optimal solution although a quite different to GRG (LINGO). But for deep more deep analysis, the total purchased product unit and the total purchased product units all of them are resulted a sufficient closed optimal solution to GRG (LINGO). To observe that, we describe the relative error value shown in Figure 3. 


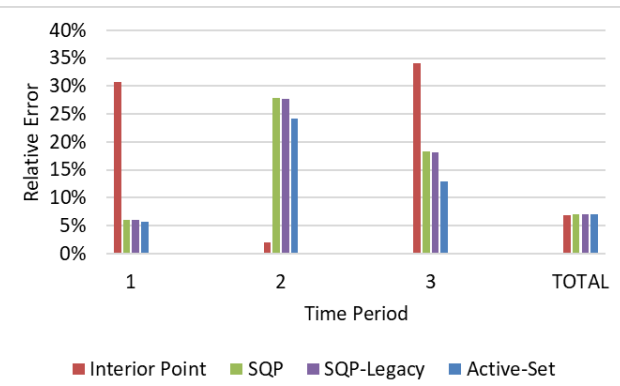

(a)

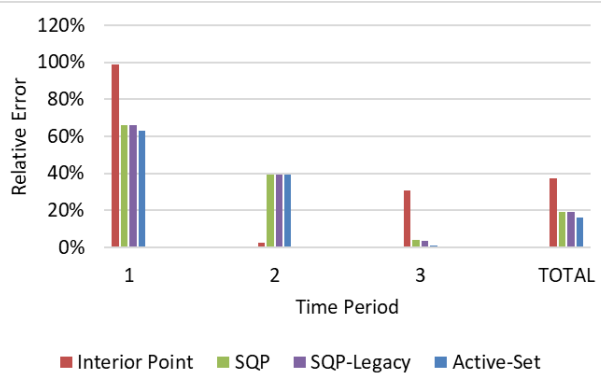

(b)

Figure 3. Relative error of interior point, SQP, SQP-legacy and active-set to LINGO solution (a) scenario-1 (b) scenario-2

These results show us that the numerical optimization interior point, SQP, SQP-legacy and active-set are all applicable to solve instead of analytical method for large scale optimization problem. Active-set was given the best relative error which means that activeset method is the best choice based on the relative error. In case to solve a large-scale problem, tt can be derived that, active-set is a best method to solve.

\section{Concluding remarks}

In this article, some classic numerical optimization algorithms which are interior point, SQO, SQP-legacy and active-set were used to solve a stochastic optimization model for supplier selection problem and inventory management. From the comparison, it can be concluded that active-set algorithm was given the better result than the others to solve a large-scale problem due that it gave the best relative error.

In our future works, we will compare the results to some meta-heuristics algorithms and analyse who will be the best method in order to find the more eligible method to solve a large-scale optimization problem in supplier selection and inventory management problem. Furthermore, we also will employ a high-performance computer to compute in order to simulate larger problem than problem solved in this paper.

\section{Acknowledgement}

Authors would like to thank FSM UNDIP for financial support under DIPA FSM UNDIP 2019 research grant.

\section{References}

1. S. Liu and L. G. Papageorgiou Comput. Chem. Eng., 119, 383 (2018).

2. F. Sahling and G. J. Hahn, Int. J. Prod. Econ., 207, 96 (2019).

3. E. Nadal-Roig, L. M. Plà-Aragonès, and A. Alonso-Ayuso, Comput. Electron. Agric., 161, 72 (2019).

4. A. Manello and G. Calabrese, J. Purch. Supply Manag., 25, 69 (2019).

5. N. Vanzetti, D. Broz, G. Corsano, and J. M. Montagna, For. Policy Econ., 97, 1 (2018).

6. M. Christopher, Logistics and Supply Chain Management, (Pearson Education, Great Britain, 2011).

7. N. R. Ware, S. P. Singh, and D. K. Banwet, Expert Syst. Appl., 41, 671 (2014). 
8. S. S. Kara, Expert Syst. Appl., 38, 2133-2139 (2011).

9. H. Hadian, A. Eshraghniaye Jahromi, and M. Soleimani, Manag. Sci. Lett., 8, 975 (2018).

10. M. Nejma, F. Zair, A. Cherkaoui, and M. Fourka, Decis. Sci. Lett., 8, 175 (2018).

11. Sutrisno, Widowati, and Solikhin, J. Phys. Conf. Ser., 725, 1 (2016).

12. J. Nocedal, S. J. Wright, and S. M. Robinson, Numerical Optimization, (Springer, New York, 1999).

13. L. Casacio, C. Lyra, A. R. L. Oliveira, and C. O. Castro, Comput. Oper. Res., 85, 129 (2017).

14. J. Gondzio, Eur. J. Oper. Res., 255, 308 (2016).

15. Z. P. Yang, J. Zhang, X. Zhu, and G. H. Lin, J. Comput. Appl. Math., 352, 382 (2019).

16. T. L. Hansen and T. L. Jensen, Signal Processing, 165, 7 (2019).

17. M. Klaučo, M. Kalúz, and M. Kvasnica, Eng. Appl. Artif. Intell., 77,1 (2019).

18. I. Temizer, M. M. Abdalla, and Z. Gürdal, Comput. Methods Appl. Mech. Eng., 276, 589 (2014).

19. J. Bleyer, Comput. Methods Appl. Mech. Eng., 330, 368 (2018).

20. M. Zhao, X. Ding, Z. Shi, Q. Yao, Y. Yuan, and R. Mo, Neurocomputing, 174, 187 (2016).

21. Y. Bin Qiao, H. Qi, Y. T. Ren, J. P. Sun, and L. M. Ruan, J. Quant. Spectrosc. Radiat. Transf., 193, 21 (2017).

22. Z. Liu, F. Forouzanfar, and Y. Zhao, J. Pet. Sci. Eng., 171, 542 (2018).

23. G. Liu, Y. Chen, Z. Xie, and X. Geng, Robot. Comput. Integr. Manuf., 51, 73 (2018).

24. H. Liao, W. Wu, and D. Fang, J. Sound Vib., 425, 301 (2018). 